73

th. 208

LA-5269-MS

INFORMAL REPORT

Energy Loss of a Relativistic Electron Beam

In a Plasma 
This report was prepared as an account of work sponsored by the United States Government. Neither the United States nor the United States Atomic Energy Commission, nor any of their employees, nor any of their contractors, subcontractors, or their employees, makes any warranty, express or implied, or assumes any legal liability or responsibility for the accuracy, completeness or usefulness of any information, apparatus, product or process disclosed, or represents that its use would not infringe privately owned rights.

In the interest of prompt distribution, this LAMS report was not edited by the Technical Information staff.

Printed in the United States of America. Available from

National Technical Information Service

U. S. Department of Commerce 5285 Port Royal Road

Springfield, Virginia 22151

Price: Printed Copy \$9:90; Microfiche $\$ 0.95$

A.OS 


\title{
Energy Loss of a Relativistic Electron Beam In a Plasma
}

\author{
by
}

\author{
R. A. Gerwin
}

This work supported by the Defense Nuclear Agency.

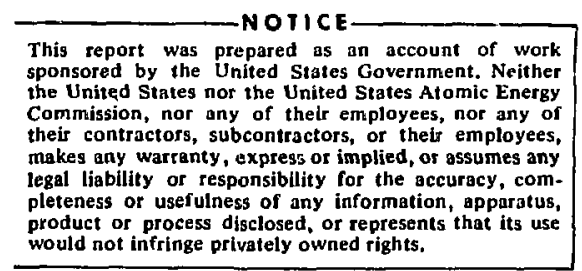


NOTATION

$n_{b}=$ density of beam electrons in the laboratory Prame

$\mathrm{k}_{\mathrm{z}}=$ axial wave-number

$c_{c}=v_{c} /\left({u k_{z}}_{z}\right)$

$u=$ beam speed

$\varepsilon=$ energy of a beam particle

$c=$ light speed

$\ell_{\mathcal{L}}=$ active length of beam involved in energy loss to Iongitudinal plasme w res

$\beta=v / c$

$Y=\left(1-\beta^{2}\right)^{-\frac{1}{2}}$

$a=$ beam radius

$L_{S}=$ characteristic slowing-down length due to energy lose to longltudinal plasme waves

$\mathrm{D}=$ beam length

$T=D / u=$ beam pulse time

$\mathrm{m}=$ electron rest mass

$\nu=\left(n_{b} \pi a^{2}\right)\left(e^{2} / m c^{2}\right)=\frac{1}{4} u_{b}^{2} a^{2} / c^{2}$

$a_{p}=$ density of plasma electrons

$\omega_{p}=\left(4 \pi m_{p} e^{2} / m\right)^{\frac{1}{2}}=$ electron plssma frequency

$x_{u}=w_{p} / u$

$\kappa_{c}=\omega_{p} / c$

$v_{c}=$ collision frequency of plasme electrons

$\ell_{c}=u / v_{c}=$ coll1sional decay length; $\nu_{c} \tau=D / l_{c}$

$\ell_{j}=\left(u / \nu_{c}\right) a^{2} k_{c}^{2}=l_{c} a^{2} k_{c}^{2}=$ decay length of plasma reverse current

$l_{3}=$ active length of beam involved in energy loas to reverse current

$\mathrm{L}_{\Im}=$ charecterist1c slowing dow length due to energy 1088 to reverse current

$\left|z_{-}\right|=$distance of en observation point behind the beam head

$z_{+}=$distance of an observetion point ahead of the beam tail

$\mathbf{P}=$ expended power

$P_{b}=1$ initial beam power 
ENERGY IOSS OF A RETATIVISTIC EHLETRON BEAM IN A PLASMA

by

R. A. Gerwin

ABSTRACT

The energy loss of a finite radiu, finite length, low density, relativistic electron beam propagating through a resistive, infinite, fieldiree plasma is calculated. The presentation 1s both more general and more quantitative then the estimate of Lovelace and Sudan [?hys. Rev. Lett. 27 (1971) 1256] who neglected the excltation of plasma osc1llations, assumed $a$ beam of Infinite extent behind the beam head, and did not utilize the exact solution of the relevent electromagnetic diffusion equation. Here, a unified calculation of the energy 1085 to both longitudinal and transverse plasma disturbances is presented wherein are incorporated the exact solutions of Mexwell's equations coup' : 1 to a linearized cold plesma model. In the appropriate parameter range, + , results are in essential agreement with the work of Lovelace and Sudan. Jut encompaas a broader paremeter range.

It 18 noted that the conditj. a usually stated for the validity of the linearized plasma dymanica, beam density $\left(n_{b}\right) \ll$ plasma density $\left(n_{p}\right)$, is insufficient. Insteed, the mucl: more restrictive condition, $n_{b} / n_{p} \ll v_{c} / w_{p}$, is required, where $w_{c}$ and $\omega_{p}$ are the plasma-electron collision frequency and plasma rrequency, respectively.

\section{INTRODUCTION}

Recent developments in eiectron beam technology have allowed the production of very high-power beans ( $10^{12}$ watts). Such beams may have a number of applications, such as the production and heating of nuclear-fusion plasmas. ${ }^{1}$

A beem traveling in vacuum not only suffers from lack of spece-charge neutrallzation, but at sufficlently high currents the beam electrons are unabie to propagate acrosa their self-magnetic

f1eld. This leads to a limiting maximum current, $I_{A}=17000$ BY amps, where $\beta=u / c, \gamma=\left(1-\beta^{2}\right)^{-\frac{1}{2}}$, with $u=$ beam speed and $c=1$ ight speed. ${ }^{2}$

It has been found that a plasma can currentneutralize as well as charge-neutrallze an electron beam, ${ }^{3}$ thus ollowing the above-mentioned currentIImitation to be overcome. Lovelace and Sudan ${ }^{4}$ pointed out that appreciable beam aras and associated plasma heating are to be expected from excitation of the plasma reverse current by high-current beams. As they point out, the importance of this mechanism 18 that the lons can be heated by the reverse-current drift velocity of plasma electrons via the Ion-acoustic Instability, whereas the beamelectron, plasma-electron two-stream instability primarily heats electrons. Iovelace and Sudan ${ }^{4}$ estimaced the beam energy 1058 due to excitation of the reverse current. They neglected the excitation of plasna oscillations, assumed an infinitely extended beam behind the beam head, and did not fully utilize the relevant electrromagnetic diffusion equation. Here, more quant1. tative results are obtained, allowing both for the excitation of plesme oscillations and for fintte length beams. The beam can easily be shorter than the reverse current decay length, so finite bearlength calculat lons are relevant. For yery long beans, the present results are in essential agreement with those of Ref. (4). 


\section{GENERAI RESULTS}

Let $P_{b}$ be the initial beam power, $n_{b}$ the bearu density as observed in the laboratory frame, a the bean radius, and define a beam density parameter

$$
v=n_{0} \pi a^{2} e^{2} /\left(m c^{2}\right)=\frac{1}{4}\left(\omega_{b}^{2} a^{2} / c^{2}\right)
$$

where if 18 the electron rest mass.

Then, the fractional power lost (hence the minus slgn) by the beam has been found to be (as derived later),

$$
P / P_{b}=-C(s+g)
$$

where

$$
c=8 \frac{\nu}{Y-I}=\frac{240}{B} \frac{I^{\prime}}{V^{\prime}}
$$

(I' is the current in amps, $V '$ the voltage in volts), with the contribution due to radiation of longltudinal waves reading

$$
\begin{aligned}
& \mathcal{L}=\left[1-e^{-\frac{1}{2} \frac{D}{l_{c}}} \cos \left(x_{u} D\right)\right] . \\
& \cdot \int_{0} x d x \frac{J_{1}^{2}(x)}{x^{2}} \frac{1}{x^{2}+a^{2} k_{u}^{2}}
\end{aligned}
$$

and the contribution due to excitation of the reverse current beling

$$
\begin{gathered}
\Im=\beta^{2} \int_{0}^{\infty} x d x \frac{J_{1}^{2}(x)}{x^{2}} \frac{a^{2} k_{c}^{2}}{x^{2}+a^{2} k_{c}^{2}} . \\
. \frac{1-\exp \left(-\frac{D}{l_{c}} \frac{x^{2}}{x^{2}+a^{2} k_{c}^{2}}\right) .}{x^{2}} .
\end{gathered}
$$

Here, $D$ 18 the bean length, $k_{u}=\omega_{p} / u$ and $k_{c}=\omega_{p} / c$ with $w_{p}$ the plasma-electron plasma frequency, and $\ell_{c}=u / v_{c}$ with $v_{c}$ the plasma-electron collision frequency. Also, $J_{1}(x)$ if $e$ Bessel function of the Plrst kind, of order un: ty. A basic assumption in the derivation of tinese en Tressions has been $\nu_{c}$ $\ll w_{p} \beta$, and precludes the J.1s j.t $\gamma \rightarrow 1(u \rightarrow 0)$ in Eq. (3).
III. EVALUUATION OF THE LONGITUDINAL RADIATION LOSS since $\int_{0}^{\infty} x^{-1} J_{1}^{2}(x) d x=\frac{1}{2}, E q$. (4) becomes, for large $a^{2} k_{u}^{2}$ which is usuelly the case,

$$
\Sigma=\left[1-e^{-\frac{1}{2} \frac{D}{l} c} \cos \left(k_{u} D\right)\right] \frac{1}{2 a^{2} k_{u}{ }^{2}} ; a^{2} k_{u}^{2} \gg 1
$$

and if $D \gg l_{c}$ es may realistically be expected, Eqs. (2) and (3) give the fractional power loss due to radiation of longitudinal plasma oscillations as

$$
C \Sigma=4 \frac{v}{\gamma-1} \frac{u^{2}}{u_{p}^{2}{ }^{2}}=\frac{n_{b}}{n_{p}} \frac{\beta^{2}}{\gamma-1} ;
$$

$$
a^{2} k_{u}^{2} \gg 1 ; D \gg l_{c} \text {, }
$$

where $n_{p}$ is the plasme electron density.

Attempts have been mede to produce millimeter beams with a Bennett cathode guide. 5 In a plasma of density $10^{14} \mathrm{~cm}^{-3}$, one would then have, when $u \sim c$, $u / w_{p} \sim a$ or $a x_{u} \sim 1$. For general velues of $a x_{u}$, one the needs to evaluate the complete Bessel function Integral of Eq. (4). This can be done approximately In terms of elementary functions, if one notes (see Fig. 1) that the domiriant range of integration 18 deternined by $\mathrm{J}_{1}{ }^{2}(x) / x^{2}$ which is fairly-well represented in th1s range by 0.28 ( $1-j x$ ). (The integration is now cut off at $x=3$ ). Then one finds, from Eq. (4),

$$
\mathcal{I}=0.28\left[1-e^{-\frac{1}{2} \frac{D}{l_{c}}} \cos \left(x_{u} p\right)\right]+\left(\frac{3}{a_{k}}\right),
$$

where the function $f(c)$ is plotted in Fig. 2, and reads

$$
\begin{aligned}
& f(\varepsilon)=\frac{1}{2} \ln \left(1+\varepsilon^{2}\right)-\left(1-\varepsilon^{-1} \arctan \varepsilon\right) .(9) \\
& \text { As } \varepsilon \rightarrow 0, f(\varepsilon) \approx \frac{2}{6} \varepsilon^{2} \text {, so the approximate }
\end{aligned}
$$

$s$ of Eq. (8) becomes $s \approx\left[1-\exp \left(-\frac{1}{2} \mathrm{D} / \mathrm{l}_{\mathrm{c}}\right) \cos \right.$ $\left.\left(k_{u} D\right)\right] 0.42\left(\varepsilon k_{u}\right)^{-2}$ for $a^{2} k_{u}^{2} \gg 1$, whlch 1s only 16 omaller than the exact expression, Eq. (6). 


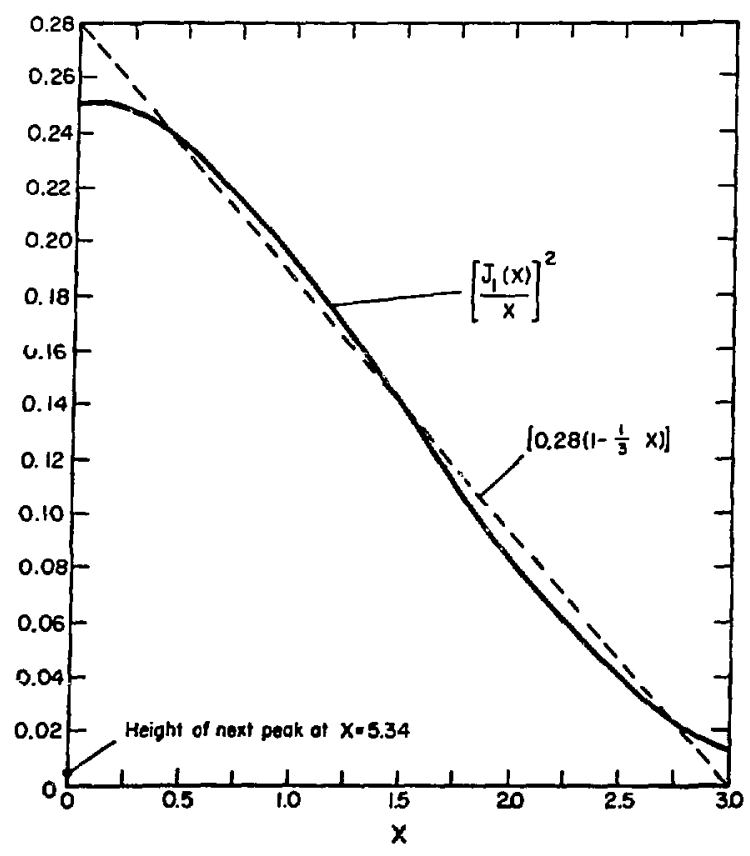

Fig. 1. An approximation for $J_{1}^{2}(x) / x^{2}$.

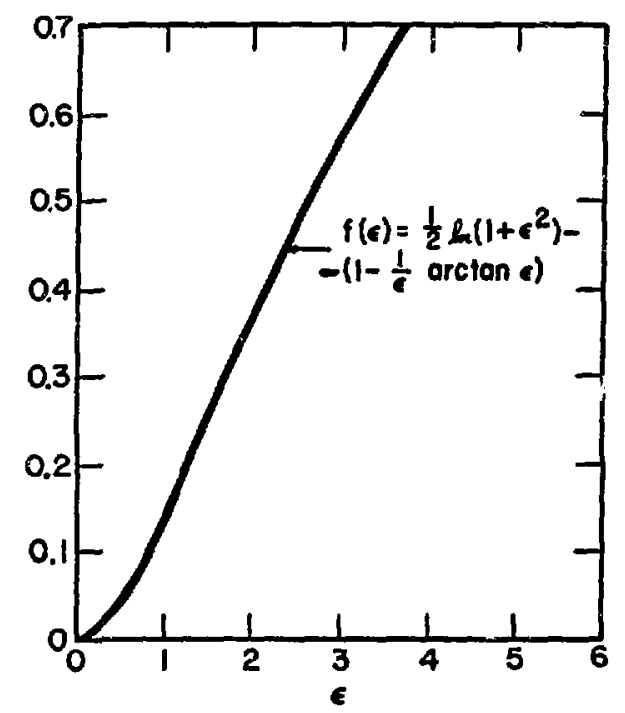

Fig. 2. An e日timate of the (akp $)$ dependence of Eq. (4). Here, $c=3 /\left(a_{x_{u}}\right)$.
Combining Eqs. (2), (3i, and (8), one has the fractional power raciated to longitudinal oscillations for $a_{k} \geqslant 1$, namely

$$
C S \approx 2.24 \frac{v}{\gamma-1}+\left(\frac{3}{2 k u}\right) .
$$

For $\varepsilon \approx 3$ or ax $u=1$, Fig. 2 gives $f(\varepsilon) \approx \frac{1}{2}$, and the fracticnal radiated power is then of order $v /(y-1)$.

As discussed later, the result for $a_{1} \approx 1$ is valid only for very small - $v$ beams.

IV. EVILUATION OF THE POWER EXPENDED IN DRTVING THE REVERSE CURRENT

A. Short Begms

Suppose $\nu_{c}=3 \times 10^{10} \mathrm{sec}^{-1}, u=c, w_{p}=20^{12}$ $\sec ^{-1}$, and $a=I \mathrm{~cm}$. Then the reverse current exisis within a length $l_{j}$ of the beam head, 3,4 where $\ell_{j}=\frac{u_{n}}{v_{c}}\left(\frac{\omega_{p}}{c}\right)^{2} \approx 10^{3} \mathrm{~cm}$ for this example. For beam pulse times $<30 \mathrm{nsec}$, one would then have $\mathrm{D}<\boldsymbol{l}_{3}$. Thus, the short-bean case is realistic.

Now consider Eq. (5) for 3 . (Note that in the absence of collisions, $\ell_{c}+\infty, 3 \rightarrow 0$, and there is no net energy loss to the reverse current. This Is because the transverse waves associated with th1s term have phase velocities larger than $c$, and so cannot be driven resonantly by the beam). From F1g. 1 , one sees that $J_{1}{ }^{2}(x) / x^{2}$ effectively cuts off the integration at $x \approx 3$. For anc $_{c} \geq 3$, the argument of the exponential in this range is then roughly $\left(-D / \ell_{g}\right)$, where $\ell_{j}=\ell_{c}\left(a^{2} \kappa_{c}^{2}\right)$. Thus, the exponentiel can be replaced by $\sim$ unity if $\mathrm{D} \ll \mathcal{L}_{j}$ which is the case we now consider. Thus, expanding the exponential, Ea. (5) becomes

$$
\begin{aligned}
& J=\beta_{a}^{2} k_{c}^{2} \frac{D}{l_{c}} \int_{0}^{\infty} x d x \frac{J_{1}^{2}(x)}{x^{2}} \\
& \frac{1}{\left(x^{2}+a_{k_{c}}^{2}\right)^{2}} ; \mathrm{D} \ll \ell_{j} \text {. } \\
& \text { When } a^{2} K_{c}^{2} \gg 1 \text {, this becomes, since } \\
& \int_{0}^{\infty} x^{-1} d x x_{1}^{2}(x)=\frac{1}{x} \text {, }
\end{aligned}
$$




$$
\Im=\frac{1}{2} a_{a^{2} k_{c}^{2}}^{\frac{g^{2}}{l_{c}}} ; a^{2} k_{c}^{2} \gg 1 ; D \ll \ell_{j}
$$

and, from Eqs. (2) and (3), the Practional power expended in driving the reverse current resds

$$
\begin{gathered}
\Theta=4 \frac{\nu}{\gamma-1} \theta^{2} \frac{D}{\ell_{j}}=\frac{q_{b}}{a_{p}} \frac{\beta^{2}}{\gamma-1} \frac{D}{\ell_{c}} ; \\
a^{2} K_{c}^{2} \gg 1 ; D \ll \ell_{j} ;
\end{gathered}
$$

since $\ell_{j}=\ell_{c} a^{2} k_{c}{ }^{2}$. Comparing with Eq. (7), one sees that the power expended by a short beam in driving the reverse current is just $\left(D / \ell_{c}\right)$ times that radiated to plasma oscillations.

To validate the assertion that Co really is the fractional power expended in driving the reverse current, note that in a steady atate, this must sust be the (fractionel) resistive power disaipated by the reverse current itself. Then, siose the current neutralization involves equal magnitude bearn $\left(\mathrm{n}_{\mathrm{b}} \mathrm{ue}\right)$ and plasme $\left(\mathrm{j}_{\mathrm{p} 1}\right)$ current densities, 3,4 one can calculate the (fractional) resiative power dissipated by the induced plasma current to be $(\sigma=$ plasma conductivity)

$$
\begin{aligned}
& \frac{J_{p 1}^{2}}{\sigma} \frac{\text { beam volume }}{\text { beam power }}=\frac{n_{b}^{2} e^{2} \beta^{2} c^{2}}{n_{p} e^{2} /\left(m v_{c}\right)} \frac{\pi a^{2} D}{n_{b} \beta c \pi a^{2}(\gamma-1) m c^{2}} \\
& \quad=\frac{n_{b} \beta^{2} D}{n_{p}(\gamma-1) l_{c}}
\end{aligned}
$$

in exact agreement with Eq. (13).

For general values of $a r_{c}$, the integral of Eq. (11) can be evaluated appraximately, as in Section III. One notes that

$$
\int_{0}^{\infty} x d x \frac{J_{1}^{2}(x)}{x^{2}} \frac{1}{\left(x^{2}+a^{2} \kappa_{c}{ }^{2}\right)^{2}}
$$

$$
\begin{aligned}
& =-\frac{d}{d\left(a^{2} k_{c}^{2}\right)}\left[\int_{0}^{\infty} x d x \frac{J_{1}^{2}(x)}{x^{2}} \frac{1}{x^{2}+a^{2} k_{c}{ }^{2}}\right] \\
& =-\frac{d}{d\left(a^{2} k_{c}^{2}\right)}\left[0.28 \rho\left(\frac{3}{a+k_{c}}\right)\right] \\
& =\frac{0.14}{a^{2} K_{c}{ }^{2}}\left[1-\frac{k_{c}}{3} \arctan \left(\frac{3}{8 k_{c}}\right)\right]
\end{aligned}
$$

where $f(\varepsilon)$ 1s the function of $B_{4}$. (9). Then Eq. (11) reads

$3 \approx 0.14 \beta^{2} \frac{D}{l_{c}}\left[1-\frac{\operatorname{arc} c}{3} \arctan \left(\frac{3}{\operatorname{akc}}\right)\right] ; D \ll \ell_{j} \cdot(14)$ Since $\left[1-\varepsilon^{-1} \arctan \varepsilon\right]+\frac{1}{3} \sigma^{2}$ as $\sigma \rightarrow 0$, the approximate $\Im$ becomes $\Im \approx 0.42 \frac{\beta^{2}}{a^{2} K_{c}{ }^{2}} \frac{D}{l_{c}}$ when $a^{2} r_{c}^{2} \gg 1$, which 1s $\approx 16 \%$ smaller than the exact expression, Eq. (12).

From Eqs. (2), (3), and (14), the power expended by short beams in driving the reverse cur rent becomes

$$
\begin{gathered}
\omega \approx 1.12 \frac{\nu}{\gamma=1} \beta^{2} \frac{D}{l_{c}}\left[1-\frac{a \epsilon_{c}}{3} \arctan \left(\frac{3}{a_{c}}\right)\right] ; \\
D \ll l_{j} .
\end{gathered}
$$

The function $\left[1-e^{-1} \arctan \epsilon\right]$ 1s plotted in Fig. 3 , which shows that, for $a_{k} \approx 1$ or $c / \omega_{p} \approx a$, corresponding to $\varepsilon \approx 3$, the fractiunal power loss is of order $\frac{2}{Y-1} \theta^{2} \frac{D}{\ell_{c}}$. Th1s case corresponds now to $D \ll \ell_{c}$, since $\ell_{c}=\ell_{\mathrm{J}}$ when $\operatorname{ax}_{\mathrm{c}}=1$, and thus involves ultra-short beam pulses. 


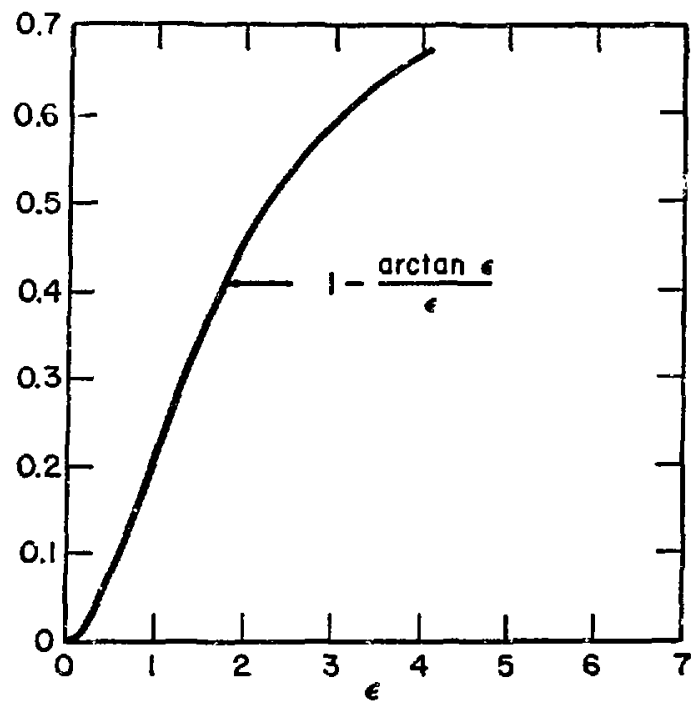

Fig. 3. Ars estimate of the $\left(a x_{c}\right)$ dependence of Eq. (11).

\section{B. Long Begme}

We now suppose that the beam length, $\vec{L}$, far exceeds the length over which the plasma reverse current exists, $\ell_{j}$. It now proves convenient to rewrite $\mathrm{Eq}$. (5) as

$$
3=3_{1}-\mathfrak{I}_{2} \text {, where }
$$

$3_{1}=\beta^{2} a^{2} k_{c}^{2}\left(\frac{1 \pm m}{\delta+0}\right) \int_{0}^{\infty} x d x \frac{J_{1}^{2}(x)}{x^{2}} \frac{1}{x^{2}\left(x^{2}+a^{2} k_{c}{ }^{2}\right)}$

and

$\Im_{2}=\beta^{2} \theta^{2} K_{c}^{2}\left(\frac{\lim }{6 \rightarrow 0}\right) \int_{\delta}^{\infty} x d x \frac{\Xi_{1}^{2}}{x^{2}}$.

$$
\frac{\exp \left\{-\frac{D}{l_{c}} \frac{x^{2}}{x^{2}+a^{2} k_{c}^{2}}\right\}}{x^{2}\left(x^{2}+a^{2} k_{c}^{2}\right)}
$$

For $a^{2} k_{c}^{2} \geqslant 1$, it is not difficult to show that the dominant term of $3_{1}$, as $\delta \rightarrow 0$, is given by [since $J_{1}^{2}(x) / x^{2} \rightarrow \frac{1}{4}$ es $x \rightarrow 0$ ]
$3_{1} \approx-\frac{1}{4} \theta^{2} \ln \delta+$ terms of order unity.

As for $\mathfrak{Y}_{2}$, the arglinent of the exponential is

$$
-\frac{D}{l_{j}} \frac{x^{2}}{1+\left(x / a_{c}\right)^{2}}
$$

so the integration is essentially completed when $x \sim \sqrt{l_{j} / D} \ll 1$, since we now suppose $D^{\frac{1}{2}} \gg \ell_{j}^{\frac{1}{2}}$. Therefore, in Eq. (18), $J_{1}{ }^{2}(x) / x^{2}$ can be replaced by $\frac{1}{4}$, and $x^{2}$ can be neglected against $a^{2} K_{c}{ }^{2}$ in the denominator since only $a^{2} K_{c}^{2} \geqslant 1$ is of interest. In the exponertial also, $x^{2}$ can be neglected against $a^{2} K_{c}^{2}$ provided that $l_{j} / D \ll a^{2} K_{c}^{2}$, which is certainly true under the assumed conditions. Then Eq. (18) becomes

$$
\int_{\left(\frac{D}{\ell_{j}} \delta^{2}\right)}^{\infty} \frac{d y}{y} e^{-y}=\frac{d}{\delta} B^{2} \frac{21 m}{\delta \rightarrow 0} E\left(\frac{D}{\ell_{j}} \delta^{2}\right)
$$$$
3_{2} \approx \frac{1}{4} \beta^{2} \frac{\lim }{\delta \rightarrow 0} \int_{\delta}^{\infty} \frac{x d x}{x^{2}} \exp \left(-\frac{D}{l_{j}} x^{2}\right)=\frac{\lambda}{\theta} \beta^{2} \frac{l i m}{\delta \rightarrow 0}
$$

where $E_{1}$ is a well-known form of the exponential integral. As $\varepsilon \rightarrow 0$, the dominant term of $E_{1}(\varepsilon)$ is - In $\varepsilon$, so $3_{2}$ becom :

$\Im_{2} \approx-\frac{1}{B} \theta^{2} \ln \left(\frac{D}{\varepsilon_{j}} \delta^{2}\right)+$ terms of order unity.

Comblining Ens. (16), (19), and (21), one has, to dominant order,

$J \approx \frac{t}{8} \beta^{2} \ln \left(\frac{D}{\ell_{j}}\right) ; \mathrm{D} \gg \ell_{j} ; a^{2} k_{c}^{2} \geqslant 1$.

Then, Eqs. (1) and (2) give the fractional. power expended in driving the reverse current as 
$\omega \approx \frac{v}{Y-I} \beta^{2} \ln \left(\frac{D}{i_{j}}\right) ; D \gg \ell_{j} ; a^{2} K_{c}^{2} \geq 1$.

This result is in essential agreement with that of Lovelace and Sudan, 4 afffering only in the logarithmic factor. They have $\ln \left(b^{2} / a^{2}\right)$ where $b$ is the outer radius of the plasma through which propagates a semi-infinfte beam. The present model, however, consists of a finite-iength bean in an unbounded plasma. It should also be noted that Eq. (23) was derived under the more general condition axc $c \geq 1$, whereas Ref. (4) used ax $\gg 1$ in the form ${ }^{P} \approx-B^{B}$, at the beam head, 1.e., complete magnetic neutralization at the beam head. (When are $\sim 1$, much of the reverse current flows outside the beam, and there 18 then little magnetic neutralization). 3

v. DERTVATION OF THE GENERAL RESULTS OF SECTION II All calculations are done in the laboratory frame, In which the m: iplane of the besun has coordinate ut. The beam head 18 at $u t+D / 2$ and the tall at ut - $\mathrm{D} / 2$. In a steady-state, oll fields, and charge and current distributions will be functions only of the combination $\vec{r}^{\prime}=\vec{r}-\vec{u} t=(\vec{R}, z-u t)$, when the beam moves along the $z$-axis.

The power lost by the beam is then

$P=u \int_{\infty} E_{z}(\vec{r}, t)_{p_{b}}(\vec{r}, t) d^{3} r=u \int_{\infty} E_{z}\left(\vec{r}^{\prime}\right) p_{p_{b}}\left(\vec{r}^{\prime}\right) d^{3} r^{\prime}$

where $\rho_{b} 1 s$ the beam charge density, and $E_{2}$ is the total axial electric fleld.

In terms of the Fourier transform

$$
v(\vec{k})=(2 \pi)^{-\frac{3}{2}} / t\left(\vec{r}^{\prime}\right) e^{-1 \vec{k} \cdot \vec{r}^{\prime}} d^{3} r^{\prime}
$$

Eq. (24) can be rewritten

$$
P=u \int_{\infty} E_{z}(\vec{k}) \rho_{b}(-\vec{k}) d^{3} k
$$

If one notes that, for $\vec{k}=\left(\vec{k}, k_{z}\right)$, the Bessel

functions obey the relations $2 \pi J_{0}(K R)=\int_{0}^{2 \pi} \exp$

$(-1 K R \cos \theta) d \theta$ and $(d / d x)\left[x J_{1}(x)\right]=x J_{0}(x)$, then the uniform electron beam charge distribution of

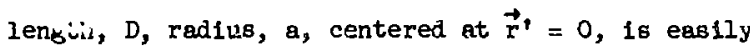
found to trensform to

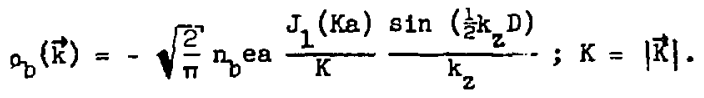

It is well known that a cold plasma model is sufficient for the study of disturbances whose characteristic length far exceed the Debye length, $\sim v_{t h} / \omega_{p}$, where $v_{t h}$ is the plasma electron thermal speed. This is certainly the case in the present problem, wherein the shortest lengths of interest prove to be $\sim c / \omega_{p}$, and the plasma is non-relativistic $\left(v_{t h} \ll c\right)$ with u $v_{\text {th }}$. Assuming that the beem only sllghtly perturbs the plasma, one then finds the transform of the exial electric field to be

$E_{z}(\vec{k})=-4 \pi i k_{z} \rho_{b}(\vec{k}) y^{2}\left(\frac{k_{z}^{2}}{D_{L} D_{T}}-\frac{\beta^{2}}{D_{T}}\right)$

where

$$
\begin{aligned}
& D_{L}=k_{z}^{2}-k_{u}^{2} /\left(1+1 \epsilon_{c}\right) \\
& D_{T}=k_{z}^{2}+\gamma^{2}\left[K^{2}+k_{c}^{2} /\left(1+1 \epsilon_{c}\right)\right] \text {, and } \\
& \varepsilon_{c}=v_{c} /\left(u k_{z}\right) .
\end{aligned}
$$

Equation (27) is derived in the Appendix.

Substitution of Eqs. (26) and (27) Into (25) produces

$$
\begin{gathered}
P=-16 \pi 1 u \gamma^{2} n_{b}^{2} e^{2} a^{2} \int_{0}^{\infty} K d K \frac{J_{1}^{2}(K a)}{K^{2}} . \\
-\int_{-\infty}^{\infty} d k_{z} \frac{s_{1}{ }^{2}\left(\frac{1}{2} k_{z} D\right)}{k_{z}}\left(\frac{k_{z}{ }^{2}}{D_{L} D_{T}}-\frac{\beta^{2}}{D_{T}}\right) .
\end{gathered}
$$

In vacuum, $\omega_{\mathrm{p}}=0$, so $D_{\mathrm{L}}=\mathrm{k}_{2}^{2}$ and $D_{\mathrm{T}}=$ $k_{2}^{2}+y^{2} K^{2}$. Then, the $k_{2}$ - Integral of Eq. (30) is $\int_{-\infty}^{\infty} d k_{z} \frac{\sin ^{2}\left(\frac{2}{2} k z^{2} D\right)}{k_{z}} \frac{1-B^{2}}{k_{z}^{2}+\gamma^{2} K^{2}}=0$ 
as it is the integral of an odd function over an even interval. Thus, the self-field of the beem produces no net energy loss, as we expect.

In collisionless plasma, for a steady state, $\omega=u_{z}$, the roots of $D_{L}$ correspond to $\omega^{2}=\omega_{p}^{2}$ and thus to excitation of longitudinal plasma oscillations. The routs of $D_{T}$ correspond to $\omega^{2}=w_{p}^{2}$ $+c^{2} k^{2}$ and thus to (non-resonent) excitation of transverse plasma disturbances. In a slightly resistive plasma (meaning essentially $v_{c} \ll \omega_{p}$ ), $D_{T}$ has an additional root corresponding to the resistive diffusion of the transverse disturbances. If one writes $\sin ^{2}\left(\frac{1}{2} k_{z} D\right)=\frac{1}{2}\left[1-\frac{1}{2}\left(e^{1 k_{z} D}\right.\right.$ $\left.\left.+e^{-1 k_{z} D}\right]\right]$ then each of the various terins of the $k_{z}$ integral of $\mathrm{Eq}$. (30) may be integrated by contour integration. The roots of $D_{L}$ are, approximately, $\mathrm{k}_{ \pm}{ }^{L} \approx \pm k_{u}-\frac{1}{2} 1 \frac{\nu_{c}}{u}$, provided $\nu_{c}{ }^{2} \ll u_{p}{ }^{2}$. The roots of $\mathrm{D}_{\mathrm{T}}$ are, approximately $\mathrm{k}_{ \pm}{ }^{\mathrm{T}} \approx \pm 1 \gamma \sqrt{K^{2}+k_{c}{ }^{2}}$ . $\frac{1}{2} 1 \frac{\nu_{c}}{u^{2}} \frac{\kappa_{c}{ }^{2}}{K^{2}+\kappa_{c}{ }^{2}}$, provided $\nu_{c} \ll \kappa_{\omega_{p}}$; and $k_{\nu} \approx-i \frac{\nu_{c}}{u} \frac{K^{2}}{K^{2}+k_{c}^{2}}$, provided $\frac{\nu_{c}^{2}}{\omega_{p}^{2}} \ll \beta^{2} \gamma^{2} \frac{a^{2} w_{p}^{2}}{c^{2}}$ or $\ell_{j}^{2} \gg \frac{c^{2}}{\gamma^{2} w_{p}^{2}}$. The latter root has to do with the resistive diffusion of the transverse waves.

The most stringent requirement on $v_{c}$ is the second one, $v_{c} \ll \beta \omega_{p}$, or $\frac{c}{w_{p}} \ll \ell_{c}$. It precludes the limit $u \rightarrow 0$ or $\gamma \rightarrow 1$.

In addition 1t should be noted that the $\beta^{2}$ term of $\mathrm{Eq}$. (30) has a pole at $\mathrm{k}_{\mathrm{z}}=0$.

The varlous roots and integration paths are displayed in FIg. 4. (The original integrand has no pole at $k_{z}=0$, and so the path can be trivially indented above the orlgin). The algebra is tedious but straightforward. When terms of order $\left(v_{c} / \omega_{p}\right)$ are neglected everywhere except in exponente, the expended power, Eq. (30), con be written

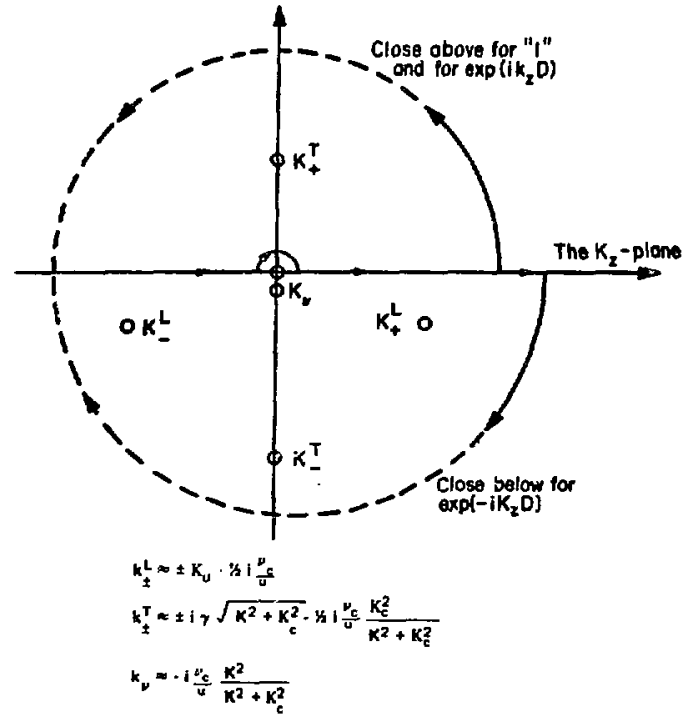

FIg. 4. The integration path and poles for the $K_{2}$ integration of the power equation, $\mathrm{Eq}$. (30).

$$
\begin{aligned}
& P=8 \pi^{2} u n_{b}{ }^{2} e^{2} e^{2} \int_{0}^{\infty} K a K \frac{J_{1}{ }^{2}(K e)}{K^{2}} . \\
& -\left\{\frac{1-\exp \left(-\frac{1}{2} \frac{D}{l_{c}}\right) \cos \left(k_{u} D\right)}{k^{2}+k_{u}{ }^{2}}\right. \\
& -\frac{B^{2} k_{c}^{2}}{K^{2}\left(K^{2}+K_{c}^{2}\right)}\left[I-\exp \left(-\frac{D}{l_{c}} \frac{K^{2}}{K^{2}+K_{c}^{2}}\right)\right]
\end{aligned}
$$

$$
\begin{aligned}
& +\frac{\left(1-\beta^{2}\right) K^{2}}{\left(K^{2}+k_{u}^{2}\right)\left(K^{2}+k_{c}^{2}\right)} \operatorname{sh}\left(\frac{1}{2} \frac{D}{\ell_{c}} \frac{k_{c}^{2}}{K^{2}+k_{c}^{2}}\right) \\
& \left.-\exp \left(-\gamma D \sqrt{K^{2}+k_{c}^{2}}\right)\right\} .
\end{aligned}
$$


When the variable of integration is changed to $x=K a$, and one divides by the beam power, $F_{b}=$ $n_{b} u \pi a^{2}(\gamma-1) m c^{2}$, then the first two terms of Eq. (31) are just those expressed by Eqs. (2) to (5). The last term (normelized to $P_{b}$ ) can be shorm to be small of order less than $\frac{v}{\gamma-I} \frac{1}{\gamma^{2} a^{2} k_{c}^{2}} \exp \left(-\gamma \frac{p}{c}\right)$ $\exp \left(\frac{1}{2} \frac{D}{l_{c}}\right)$, and will generally be negligible compared to the terms remaining, since $c / \omega_{p} \ll L$ and $v_{c} \ll \mu_{p}$ (Recall that $\ell_{c}=u / v_{c}$ ).

\section{CHAFGE AND CURRENT NEUTRALIZATION}

Although charge and current neutralization of the beam by the plasma have been discussed elsewhere, ${ }^{3}$ a brief account is presented here for completeness.

\section{A. Charge Neutralization}

The quantity $1 \vec{k} \cdot \vec{E}(\vec{k})$ is rurnished by Eq. $(A-6)$ of the Appendix. Taking the inverse transform, $\psi^{\left(\vec{r}^{\prime}\right)}=(2 \pi)^{-\frac{3}{a}} \int_{t}(\vec{k}) e^{1 \vec{k} \cdot \vec{r}^{\prime}} d^{3} k$, and applying Eq. (26) for $p_{b}(\vec{k})$, one finds

$\nabla \cdot \vec{E}=4 \pi\left(p_{\text {plasma }}+p_{b}\right)=-\frac{4 n_{b} e a}{21} \int_{d K J_{0}(K R) J_{1}(K a)}^{\infty}$

$$
\cdot \int_{-\infty}^{\infty} d k_{z} \frac{k_{z}+1 v_{c} / u}{k_{z}^{2}\left(1+1 \epsilon_{c}\right)-k_{u}^{2}}\left(e^{1 k_{z} z_{+}}-e^{1 k_{z} z}\right)
$$

where $\varepsilon_{\mathrm{c}}=v_{\mathrm{c}} /\left(\mathrm{uk}_{\mathrm{z}}\right)$, and

$\mathrm{Z}_{ \pm}=\mathrm{Z}^{\prime} \pm \frac{1}{2} \mathrm{D}=$ (signed' distance of observation point from beam $\left\{\begin{array}{l}\text { tail } \\ \text { head }\end{array}\right\}$.

The $k_{z}$ poles are in the lower half-plane, so the $e^{1 k z^{2}+}$ term gives no contribution. Since $z$. $<0$ within the beam, the integration of the $\exp \left(1 k_{z} z_{-}\right) t \in x m$ is closed in the lower half-plane. Then, using $\int_{0}^{\infty} J_{0}(K R) J_{1}(K a) d K=\left\{\begin{array}{l}a^{-1} \\ 0\end{array}\right\}$ for $R>a$, and neglecting terme of order $\left(v_{c} / w_{p}\right)$ except in exponents, one finds that Eq. (32) becomes

$o_{\text {plasma }}+q_{b}=-n_{b} e \exp \left(-\frac{1}{2} \frac{\left|z_{-}\right|}{\ell_{c}}\right) \cos \left(k_{u_{-}} z_{-}\right)$; $\operatorname{trlth} \ell_{\mathrm{c}}=u / v_{\mathrm{c}}, \kappa_{\mathrm{u}}=\omega_{\mathrm{p}} / \mathrm{u}$

This equation shows that the beam only becomes charge-neutralized beyond a distance $2 l_{c}$ behind the beam head. closer to the head, there exist plasma oscillations involving plasme density fluctuations as large as $2 \mathrm{n}_{b}$.

B. Current Neutralization

The Fourler Inversion of the exial electric field, Eq. (27), may be performed by the usual methods. The roots are as shown in Fig. 4, except that there is no longer a pole at $k_{z}=0$. After eubstitution of Eq. (26) for $p_{b}(\vec{k})$ into Eq. (27), and applying contour integration to the $k_{z}$ integral, one finds (note $z_{-}<0$ )

$$
\begin{aligned}
& E_{z}\left(\vec{r}^{\prime}\right)=4 \pi m_{b} e a \int_{0}^{\infty} d K J_{0}(K R) J_{l}(K a) . \\
& \left\{\frac{\kappa_{u}}{K^{2}+\kappa_{u}^{2}} \exp \left(\frac{v_{c}}{2 u} z\right) \sin \left(\kappa_{u^{2}}\right)\right. \\
& +\frac{B^{2}\left(v_{c} / u\right) k_{c}^{2}}{\left(K^{2}+k_{c}^{2}\right)^{2}} \exp \left(z-\frac{v_{c}}{u} \frac{K^{2}}{K^{2}+\kappa_{c}^{2}}\right)
\end{aligned}
$$$$
+\frac{\left(1-\beta^{2}\right) \kappa^{2}}{\left(\kappa^{2}+\kappa_{u}^{2}\right)\left(K^{2}+k_{c}^{2}\right)} \frac{\gamma \sqrt{K^{2}+\kappa_{c}^{2}}}{2} .
$$

$$
\begin{aligned}
& {\left[\exp \left(-z+\left(v \sqrt{K^{2}+k_{c}^{2}}-\frac{1}{2} \frac{u_{c}}{u} \frac{\kappa_{c}^{2}}{\kappa^{2}+k_{c}^{2}}\right)\right)\right.} \\
& \left.-\exp \left(z-\left(\gamma \sqrt{K^{2}+k_{c}^{2}}+\frac{1}{2} \frac{u_{c}}{u} \frac{\kappa_{c}^{2}}{K^{2}+k_{c}^{2}}\right)\right)\right],
\end{aligned}
$$

provided terms of order $\left(v_{c} / \omega_{p}\right)$ are neglected except in exponents.

The final tern (in square brackets) may be shown to contribute an electric fleld small of order less than

$\frac{4 m_{b} e}{m_{c}} \exp \left(-\left|z_{ \pm}\right| \gamma \frac{w_{p}}{c}\right) \exp \left(\frac{1}{2} \frac{\nu_{c}}{u} z_{+}\right)$ 
In which the positive exponential applies only to the $z_{+}$contribution. Thus, this term is negligible more than an electromagnetic skin depth array from the ends of the beam.

$$
\begin{aligned}
& \text { In the limit } a^{2} k_{u}{ }^{2} \gg 1 \text {, noting } \\
& \int_{0}^{\infty} d K J_{0}(K R) J_{1}(K a)=a^{-1}
\end{aligned}
$$

for $R<a$, the flrst term of $\mathrm{Eq}$. (35) reade

$$
\begin{gathered}
F_{z z} \approx-\frac{4 \pi m_{b} e}{k_{u}} \exp \left(\frac{v_{c}}{2 u} z_{-}\right) \sin \left(k_{u}^{2}\right) ; R<a ; \\
a^{2} k_{u}^{2} \gg 1 ;
\end{gathered}
$$

and Is associated with the transient plasma oscillations near the bean head.

The argument of the exponential. In the second term of Eq. (35) 15 of order $\left(-\left|z_{-}\right| / \ell_{j}\right)$ where $\ell_{j}=$ $\ell_{c} a^{2} K_{c}^{2}$ is the characteristic decay length of the plasme reverse current. Well within $\ell_{j}$ of the beam head, the exponential may be replaced by unity, and In the Iimit $a^{2} k_{c}{ }^{2} \gg 1$, one finds $\left(\ell_{c}=u / v_{c}\right)$,

$$
\begin{aligned}
& E_{a z} \approx \frac{4 m_{b} e \beta^{2}}{l_{c} k_{c}^{2}} ; R<a ; a^{2} k_{c}^{2} \gg 1 ; \\
& \left|z_{-}\right| \ll l_{g} .
\end{aligned}
$$

Far beyond the distance $\ell_{j}$ from the beem heed, $\left|z_{-}\right| \gg l_{j}$, the exponential becomes very small and the integration is essentially completed for $K \sim a^{-I}\left(e_{y} /\left|z_{-}\right|\right)^{\frac{1}{2}}$, then, one can replace $J_{0}(K R) J_{1}$ (Ka) by $\left(\frac{1}{2} \mathrm{Ka}\right.$ ) in Eq. (35), and can neglect $K^{2}$ against $k_{c}^{2}$ for $a_{k}>1$. Then the second term of Eq. (35) becomes

$$
\begin{aligned}
& E_{2 z} \approx\left(\frac{4 m_{b} e \beta^{2}}{\ell_{c} k_{c}^{2}}\right)\left(\frac{l_{j}}{4 \bar{z}^{2}-1}\right) \\
& =\frac{m_{b} e g^{2} a^{2}}{\mid z-1} ; R \leqslant a ; a k_{c} \geq 1 ;|z| \gg l_{j} \text {. }
\end{aligned}
$$

Beyond $2 \ell_{c}$ from the beam head, the transient plasma oscillations have died out, and the linsarized plasma-electron equation of motion,

$$
\left(\partial / \partial t+v_{c}\right) v_{z}=-\frac{e}{m} E_{z}
$$

reduces to

$$
v_{z}=-\frac{e}{m v_{c}} E_{z}
$$

Substitution of Eq. (37) 1nto Eq. (40) 1mmedlately ylelds the reverse current, $v_{2}=-\left(n_{b} / n_{p}\right) u$ for $\left|z_{-}\right| \ll \ell_{j}$. For $\left|z_{-}\right| \gg \ell_{j}$, substitution of $\mathrm{E}_{\mathrm{q}}$.

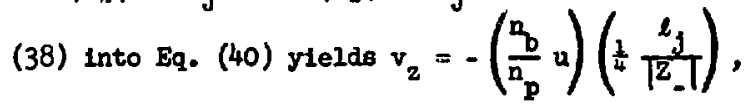
that 1s, algebrasc decay of the reverse current.

\section{DISCUSSION}

\section{A. The Energy Ioss}

General expressions [Eqs. (2) to (5)] were derived for the expended beam power in a resistive plasme, allowing for the excitation of plasma $08 \mathrm{cil}$ lations, finite beam length, finite beem radius, and allowing for the possibility of thin beams, $a \sim c / u_{p}$. These general expressions incorporate the exact, solutions of Maxwell's equations coupled to the linearized dynamics of a cold plasma. Specific evaluation of these expresaiong produced the folluwing results for the expended power, when $a \gg c / w_{p}$ and $y \gg 1$. The power radiated into plasma oscillations is $P_{c}=4 \frac{v}{y_{w_{p}}} \frac{c^{2}}{w^{2}} P_{b}$, provided $D \gg \ell_{c}$. When $D \ll \ell_{j}$, the power expended in driving the reverse current is $P_{3}=4 \frac{\nu}{\gamma} \frac{D}{l_{j}} P_{b}=\frac{D}{l_{c}}$ times the power radiated into plasma osciliations. When $D \gg l_{j}$, the power expended in driving the reverse current is $P_{j}=\frac{v}{\gamma}$ In $\left(\frac{D}{l_{j}}\right) P_{b}$, in essential agreement w1 th the work of Lovelace and Sudan. ${ }^{4}$ In addition, it was checked that the expended power is just the ohmic power dissipated by the reverse current, which 18 to be expected in $\&$ steady state.

For very thin beams, $a \sim c / u_{p}, \ell_{j} \rightarrow \ell_{c}$, these results remain as stated above, but with somewhat different numerical factors [except for $P_{j}\left(D \gg \ell_{j}\right)$ 
which is valid as is]. However, due to the use of the linearized equation of motion for the plasme electrons, the thin-beam results apply only to very small $v$ bears, as discussed jelow.

\section{B. The Restrictions of Linearized Theory}

In the Appendix, the plasme current density was approximated by $-\mathrm{en}_{\mathrm{p}} \overrightarrow{\mathrm{v}}$. Thus, the excited plasma density fluctuations were assumed small against $n_{p}$. But, according to Eq. (34), these fluctuations are of order $n_{b}$. Therefore, the assertion that $\vec{J}_{\text {plasma }}$ $=-e n_{p} \vec{v}$ requires $n_{b} \ll n_{p}$. This restriction of the linearized theory has been noted elsewhere. ${ }^{3}$ It can also be written $\nu \ll \frac{1}{4} \omega_{p}{ }^{2} a^{2} / \mathrm{c}^{2}$, see $\mathrm{Eq}$. (1).

Similar restrictions are obtained when the equation of motion (39) is considered. In the transient region, the dominant velocity term, $\partial v_{z} / \partial t$, is of the crder $w_{p} v_{z}$, and the neglected velocity terms are $v_{z} \partial v_{z} / \partial_{z}, v_{R} \partial v_{z} / \partial_{R}$, and $\omega_{c}$ net $v_{R}$, the last two occurring only in $a$ thin tube of thickness $\sim \mathrm{c} / w_{p}$ around $R=a$, wherein $v_{R} \sim v_{z} \cdot 3$ If one notes that $v_{z} \sim n_{b} c / n_{p}$, that the characteristic lengths are of order $c / w_{p}$, and that $w_{c}$ net $=e B$ net $/ \mathrm{mc}$ is the cyclotron frequency in the magnetic field of the net current, $I_{\text {net }}^{(R=a)} \sim I_{b} /\left(w_{p} \theta / c\right),{ }^{3}$ then one finds again that the neglected terms are of order $\left(n_{b} / n_{p}\right)$ compared to the dominant terms. But, these neglected terms should also be small even against $v_{c} v_{z}$, and this leads to the more restrictive irequality $n_{b} \ll$ $n_{p} \frac{\nu_{c}}{\omega_{p}}$, or $\nu \ll \frac{1}{4}\left(\frac{\omega_{p}{ }^{a}}{c}\right)^{2}\left(\frac{\nu_{c}}{\omega_{p}}\right)$.

When the transients have dled away, further then $2 \varepsilon_{c}$ from 'he geam head, the $\partial_{t} v_{z} \sim \omega_{p} v_{z}$ term is effectively no longer present in $\mathrm{Eq}$. (39). Then, the neglected tern.s must be compared to $v_{c} v_{z}$ only. In this region, the above-mentioned $v_{R}$ terms have aled away too, ${ }^{3}$ but one still has to compare $v_{z} \partial v_{z} /$ $\partial_{z}$ against $v_{c} v_{z}$. Now, however, the transients are absent, and so the characteristic length is $\sim \ell_{j}$ rather then $c / w_{p}$. Recalling that $\ell_{j}=\frac{\underline{u}}{v_{c}} \frac{\omega_{p}^{2} a^{2}}{c^{2}}$, one finds that $\left|v_{z} \partial v_{z} / \partial_{z}\right| \ll v_{c} / v_{z}$ only requires $n_{b} \ll n_{p}\left(\omega_{p} a / c\right)^{2}$ in the post-transient region. of course, this is superseded by the more restrictive inequality $n_{b} \ll n_{p}$, required everywhere, since the plasme density fluctuations were everywhere neglected against $n_{p}$ as stated above.
To summarize, it has been found that the usualIy stated condition $n_{b} \ll n_{p}$, or $v \ll \frac{1}{4}\left(\omega_{p} q / c\right)^{2}$, is insufficient for the application of the linearized theory to the transient region. What appears to be required is the much more restrictive $n_{b} \ll n_{p}$ times $\left(\nu_{c} / \omega_{p}\right)$, or $\nu \ll \frac{1}{4}\left(\omega_{p} a / c\right)^{2}\left(\nu_{c} / \omega_{p}\right)$. (However, beyond the transient region, $n_{b} \ll n_{p}$ again appears to be sufficient). When $a \rightarrow c / w_{p}$, this requires a very smell value of $v$ Indeed, namely $v \ll \frac{1}{4}\left(v_{c} / \omega_{p}\right)$. c. Slowing-Doum Ranges

It is natural to ask how far the beam travels before losing a significant fraction of its energy, 1.e., what is the range of the beam. The fractional expended power 18 directly interpretable in terms of the range. Let $\ell$ be the length of beam involved in a given energy loss mechanism. Then, assuming all particles in the volume $\pi a^{2} l$ lose energy at equal rates, one has ( $\varepsilon=$ single particle energy)

$$
\frac{P}{P_{b}} \approx \frac{n_{b} \pi a^{2} \ell\left(d_{\varepsilon} / d t\right)}{n_{b} u_{\pi a^{2}}{ }^{2}}=\frac{\ell}{u_{\varepsilon}} u \frac{d_{\varepsilon}}{d z}=\frac{\ell}{I}
$$

where the range $I$ of an affected beam particle is defined as

$$
\mathrm{L}=\epsilon /(\mathrm{d} \epsilon / \mathrm{dz})
$$

Since $\left(P / P_{b}\right)$ is known, $L$ cen be found if one knows $l$. 1. Range Due to Excitation of Plasma Osc1llations. Since alternate layers of beam of thickness $\sim \mathrm{u} / \mathrm{u}_{\mathrm{p}}$ are losing and gaining energy, the active length here is effectively $\ell_{\Sigma} \sim u / u_{p}$. In fact, using Eq. (36), the net fractional radiated power may be estimated as

$$
\begin{aligned}
\left(P / P_{b}\right)_{L} & \sim \frac{n_{b} \pi a^{2} \ell_{L} e E_{1 z} u}{n_{b} \pi a^{2} u(y-1) m c^{2}} \sim \frac{\ell_{c} e E_{1 z}}{(\gamma-1) m c^{2}} \\
& \sim \frac{\left(u / \omega_{p}\right) e\left(4 \pi n_{b} e / k_{u}\right)}{(\gamma-1) m c^{2}} \sim \frac{{w_{b}}^{2} \beta^{2}}{(\gamma-1) \omega_{p}^{2}}
\end{aligned}
$$

In egreement with Eq. (7). This shows that $\ell_{\mathcal{L}} \sim$ $u / u_{p}$ is indeed the effective active beam length for this perticular loss mechantsm. Then, from Eqs. (41) and (7) the range of the affected beam particles is 
given by

$$
L_{S} \approx l_{\mathcal{L}} /\left(P / P_{b}\right)_{\mathcal{L}} \approx \frac{\left(u / w_{p}\right)}{\left(\frac{n_{b}}{n_{p}} \frac{\beta^{2}}{\gamma-1}\right)} * \frac{\left(c / w_{p}\right)}{\left(\frac{n_{b}}{m_{p}}\right)}
$$

for $a$ highly relativistic beam in the last expression, or elso

$$
I_{\mathcal{L}} \approx \frac{\left(u / w_{p}\right)}{\left(2 n_{p} / n_{p}\right)}
$$

In the non-relativiatic limit. (Strictly speaking, this limit is forbidden by the assumptions).

2. Range Due to Driving the Reverse Current. For a short beam $\left(D \ll \ell_{j}\right)$, it 1 a clear that the entire beam is involved in driving the reverse current so $h_{3} \approx D$. In fact, using Eq. (37),

$$
\begin{aligned}
& \left(P / P_{b}\right)_{\Im} \sim \frac{\left.n_{b} \pi a^{2} l\right\} e E_{2 z} L}{n_{b} \pi e^{2} u(\gamma-1) m c^{2}}=\frac{l_{j} e E_{2 z}}{(\gamma-1) m c^{2}} \\
& =\frac{\operatorname{De}\left(4 m m_{b} e \beta^{2} / l_{c} k_{c}^{2}\right)}{(y-1) m c^{2}}=\frac{D}{l_{c}} \frac{\omega_{b}{ }^{2} \beta^{2}}{\omega_{p}^{2}(\gamma-1)}
\end{aligned}
$$

in agreement with Eq. (13). This verifies that, for a short beam the active beam length for this mechanism is indeed $\ln \approx D$. Then, using Eqs. (13) and (41), the range 15 given by

$$
\begin{aligned}
s_{j} & \approx b_{j} /\left(P / P_{b}\right)_{j}=\frac{D}{\left(\frac{n_{b}}{n_{p}} \frac{\beta^{2}}{\gamma-1} \frac{i}{l_{c}}\right)}=\frac{l_{c}}{\left(\frac{n_{b}}{n_{p}} \frac{\beta^{2}}{\gamma-1}\right)} \\
& \approx \frac{l_{c}}{\frac{n_{b}}{n_{p}}} ;\left(D \ll l_{j}\right)
\end{aligned}
$$

for a highiy relativistic beam in the final expression; or also

$$
I_{3} \approx \frac{\ell_{c}}{\left(2 n_{b} / n_{p}\right)} ;\left(D \ll \ell_{j}\right) ;
$$

In the non-relativistic limft.

For a long bean $\left(D \ll \ell_{j}\right), 1$ is is clear that the affected beam length $18 \mathrm{l}_{j} \approx l_{j}$. In fact, again ueing Eq. (37), and recalling that $\ell_{j}=\ell_{c}\left(\omega_{p} a / c\right)^{2}$,

$$
\begin{aligned}
\left(P / P_{b}\right) & \sim \frac{l_{3} e E_{2 z}}{(\gamma-1) m c^{2}}=\frac{\ell_{j} e\left(4 m m_{b} e \beta^{2} / l_{c} k_{c}^{2}\right)}{(\gamma-1) m c^{2}} \\
& =\frac{u_{b}^{2} \beta^{2}\left(w_{p} q / c\right)^{2}}{(\gamma-1)_{\omega_{p}}^{2}}=\frac{4 u \beta^{2}}{\gamma-1}
\end{aligned}
$$

In essent1el agreement with Eq. (23) except for the logarithmic factor. [Here, we have used Eq. (1) for v]. This verifles that, for a lons beam, the active beam length for this mechanism is indeed $\ell_{j} \approx \ell_{j}$. The two results w11l agree exactly if we tyke $h_{3}=$ $\frac{1}{4} x_{j} \ln \left(\frac{D}{l_{j}}\right)$. Then, using Eqs. (23) and (4I), the range is

$$
\begin{aligned}
& I_{3} \approx l_{j} /\left(P / P_{b}\right)_{y}=\frac{\frac{1}{4} \ell_{j} \ln \left(\frac{D}{\ell_{j}}\right)}{\frac{\nu}{y-1} B^{2} \ln \left(\frac{D}{\ell_{j}}\right)} \\
& =\frac{\frac{1}{4} \ell_{c}\left(\omega_{p} a / c\right)^{2}}{\frac{1}{4} \frac{\left(\omega_{b}{ }^{2} / c^{2}\right)}{\gamma-1} B^{2}}=\frac{\ell_{c}}{\left(\frac{n_{b} \beta^{2}}{(\gamma-1) n_{p}}\right)} ; \gg \ell_{j} ;
\end{aligned}
$$

in agreement with the short beam result, Eq. (45). It 18 also useful to note that Eqs. (45) and (47) can be written

$$
\operatorname{Lg} \approx \frac{\ell_{1}}{[4 \nu /(y-1)]}
$$

which is the bas1s of the statement that high $v / Y$ beans $w 11$ be stopped in a distance short compar 3d to $\ell_{j}$.

3. Comparison H1th the Two-Stream Inatab121ty. Consider first a non-relativistic beam. The two-stream instability growth rate $1 s^{6} \delta_{1} \sim \omega_{p}\left(\frac{n_{p}}{n_{p}}\right)^{\frac{1}{3}}$ corresponding to a range $L_{t . s}^{(I)} \sim \frac{\left(u / \omega_{p}\right)}{\left(n_{b} / n_{p}\right)^{\frac{5}{3}}}$ wh1ch 1a 
much shorter than Eq. (44). However, after a few growth times, the beam is s sared in axial velocity, and the quasi-linear ragime takes over, wherein ${ }^{7}$

$6_{2} \sim w_{p}\left(n_{b} / n_{p}\right)$. Then, the renge is $\tau_{t .5 .}^{(2)} \sim \frac{\left(u / w_{p}\right)}{\left(n_{b} / n_{p}\right)}$ which is of the same order as Eq. (44). In either case, the two-stream ranges, as well as the range given by Eq. (44), are much shorter than the range $I_{3}$ for driving the reverse current, because of the condition $v_{c} \ll \omega_{p}$.

It ahould also be noted that the range $I_{t . s}$. applies to all the beam electrons, whereas $\mathrm{I}_{\mathcal{L}}$ applles to oniy those within $\sim l_{c}$ of the beam head, and $I_{j}$ applies to those within $\ell_{j}$ of the beam head (for a short bean, this would involve all the beam electrons).

Consider next a relativistic beam. The initial growth rate is now ${ }^{8} \delta_{1} \sim \mu_{p}\left(n_{p} / v_{p}\right)^{\frac{3}{3}}$ corresponding to a renge $L_{t . s .}^{(1)} \sim \frac{\left(c / w_{p}\right)}{\left(n_{p} / m_{p}\right)^{\frac{3}{3}}}$ which is again much shorter then Eq. (43). Because of the large longltudinal mass of the relativiatic beam particles, exial velocity smearing is inetfective. Transverse velocity smearing leads to the two-stream growth rate in the quasi-linear regime ${ }^{8} \delta_{2} \sim$ $u_{p}\left(\frac{n_{b}}{n_{p}}\right)^{\frac{1}{3}}$, which is not too different from $\sigma_{1}$. This gives a razge $L_{t .8 .}^{(2)} \sim \frac{\gamma c / u_{p}}{\left(n_{b} / n_{p}\right)^{\frac{3}{3}}}$ wh1ch 1s again much shorter than Eq. (43). Reference (8) points out that the relativistic beam loses a significant fraction of 1 ts energy elready during the $\delta_{1}$ (hydrodymanic) phase.

In summary, the two-stream electron-electron process appears to be a significantly more effec$t$ tve energy loss mechaniom than either of the processes represented by Eqs. (43) or (45), contrary to the assertion of Lovelace and Sudan. ${ }^{4}$ Nevertheless, the energy loss to the traneverse current remains important because of the possibility of heating the plasma Ions when the reverse-current drift velocity exceeds the ion scoustic speed.

\section{ACKNOWLEDGMENTS}

The author benefited from enlightening discussions with D. Hammer, R. Sudan, and J. Freldberg.

\section{REFERENCES}

1. Sidney Putnam, "Theoretical Studies of Intense Relativiat1c Electron Beam-Plasme Interactions," Physics International Company report PIFR-72105 (DNA 2849F) (July 1972).

2. Hannes Alfuen, "On the Motion of Cosmic Rays in Interstellar Space, " Phys. Rev. 55, 425-429

(1939).

J. D. Lawson, "On the Adlabatic Self-Constriction of an Accelerated Electron Beam NeutralIzed by Positive Ions," J. Electronics and Control 3, 587-594 (1957).

J. D. Lawson, "Ferveance and the Bennett PInch Relation in Partially Neutralized Electron Beams," J. Electrontcs and Control 2, 146-151 (1958).

3. D. A. Hemer and N. Rostoker, "Propegation of High Current Relativistic Electron Beams," Phys. Fluids 13, 1831-1850 (1970).

R. Lee and R. N. Sudan, "Return Current Induced by a Relativistic Beam Propagating in a Magnetized Plasme," Phys. Fluids 14, 1213-1225 (1971).

4. R. V. Lovelace and R. N. Sudan, "Plasma Heating by High-Current Relativistic Blect,ron Beams," Phys. Rev. Letters 27, 1256-1259 (1971).

5. D. L. Morrow, J. D. Fhillips, R. M. Stringfield Jr., W. O. Doggett, and W. H. Bennett, "Concentration and Guidance of Intense Relativiatic Blectron Beans," Appl. Phys. Letters 19, 441443 (1971).

6. v. N. Tsytovich, Nonlinear Effects in Plasme (Plenum Press, New York, 1970), p. 185.

7. V. N. Tsytovich, Nonlinear Effects in Plasme (Plenum Press, New York, 1970), p. 176.

8. Ya. B. Falnberg, V. C. Shapiro, and V. I. Shevchenko, "Nonlinear Theory of Interaction Between a 'Monochromatic' Beam of Relativistic Electrons and a Plasma," Sov. Phys. -JETP 30 , 528-533 (1970). Zh. Eksp. Teor. Fiz. 57, $966-$ 977 (1969). 


\section{APPENDIX}

FOURIER TRANSFORM OF THE AXIAL EMECTRIC FIETD

If one eliminates the magnetic field from

Maxwell's equations, one has, for the electric field,

$\nabla \times \nabla \times \vec{E}+\frac{1}{c^{2}} \frac{\partial^{2}}{\partial t^{2}} \vec{E}=-\frac{4 \pi}{c} \frac{\partial}{\partial t} \vec{J}_{\text {tot }}$

where $\vec{J}_{\text {tot }} \equiv \vec{J}_{\text {bean }}+\vec{J}_{\text {plasma }}$ is the total current density.

The linearized equation of motion for a plasma electron's velocity reads

$$
\left(\frac{\partial}{\partial t}+v_{c}\right) \vec{v}=-\frac{e}{m} \vec{E}
$$

(where $\nu_{c}$ is the plasma-electron collision frequency) which is equivalent to the following equation for the plasma current density,

$$
\left(\frac{\partial}{\partial t}+v_{c}\right) \vec{J}_{\mathrm{pl} .}=\frac{w_{p}^{2}}{4 \pi} \vec{E} .
$$

In a steady state, wherein all quantities depend only on the combination $\vec{r}^{\prime}=\vec{r}-\vec{u} t=(\vec{R}, z-$ ut), one can replece $\nabla \equiv \nabla_{\vec{r}}$ by $\nabla^{\prime} \equiv \nabla_{\vec{r}^{\prime}}$, and further $\frac{\partial}{\partial t}=-u \frac{\partial}{\partial z}$. Then, one Fourler-transforms Eqs. (Ai) and $(A-3)$ with respect to $\vec{r}^{\prime}$, 1.e.,

$\psi(\vec{k}) \equiv(2 \pi)^{-\frac{3}{2}} \int d^{3} r^{\prime} e^{-1 \vec{k} \cdot \vec{r}^{\prime}} \psi\left(\vec{r}^{\prime}\right)$. Then they read

$$
\begin{aligned}
& k^{2} \overrightarrow{\mathrm{E}}-\overrightarrow{\mathrm{k}}(\overrightarrow{\mathrm{k}} \cdot \overrightarrow{\mathrm{E}})-\beta^{2} \mathrm{k}_{2}{ }^{2 \overrightarrow{\mathrm{E}}}=\frac{4 \pi u}{c^{2}} 1 \mathrm{k}_{\mathrm{z}}\left(\vec{\jmath}_{\mathrm{pl}}+\vec{J}_{\mathrm{b}}\right) \\
& \left(-u f \mathrm{k}_{\mathrm{z}}+v_{\mathrm{c}}\right)\left(4 \pi \vec{J}_{\mathrm{pl} .}\right)=\omega_{\mathrm{p}}^{2} \overrightarrow{\mathrm{E}},
\end{aligned}
$$

where $\beta \equiv u / c$.

If one takes $\vec{k}$. of Eqs. $(A-4)$ and (A-5), the following expression for $\vec{k} \cdot \vec{E}$ can be obtained,

$\vec{k} \cdot \vec{E}=-\frac{4 \pi 1 k_{z}{ }^{2} \rho_{b}(\vec{k})}{k_{z}{ }^{2}-k_{u}{ }^{2} /\left(1+i \epsilon_{c}\right)} \equiv-\frac{4 \pi I k_{z}{ }^{2} \rho_{b}(\vec{k})}{D_{L}}$

where $\kappa_{u} \equiv \omega_{\mathrm{p}} / u, \varepsilon_{c} \equiv \nu_{c} /\left(u_{\mathrm{z}}\right)$, and we have used $J_{b}=2 j_{b 2}=2 u_{p_{b}}$.

One cen now take the axial components of Eqs. (A-4) and (A-5), and, with the help of Eq. (A-6) can find $E_{z}(\vec{k})$. The result is

$$
E_{z}(\vec{k})=-4 \pi 1 k_{z} \gamma^{2} \rho_{b}(\vec{k})\left\{\frac{k_{z}^{2}}{D_{L}}-\beta^{2}\right\} \frac{l}{D_{T}}
$$

where

$D_{T} \equiv k_{z}^{2}+\gamma^{2}\left(K^{2}+k_{c}^{2} /\left(1+1 \varepsilon_{c}\right)\right)$,

and $\gamma \equiv\left(1-\beta^{2}\right)^{-\frac{1}{2}}$, with $\theta \equiv u / c$. 